\section{Taking the inflammation out of epilepsy}

\section{By Tim Fulmer, Senior Writer}

Italian and U.S. researchers have proposed treating epileptic seizures by targeting an inflammatory pathway outside the blood-brain barrier: leukocyte migration and recruitment. ${ }^{1}$ The strategy potentially could help patients whose seizures are not adequately controlled by existing epilepsy drugs, while also avoiding the psychiatric side effects of those treatments.

Epilepsy drugs that target receptors in the brain are not always successful at blocking seizures, suggesting they may not address the underlying cause of disease in some patients. Moreover, many of the drugs are associated with psychiatric side effects. Indeed, last month, the FDA started requiring antiepileptic drug labels to warn of increased risk of suicidality.

The new findings could lead to the repurposing of several inflammation therapeutics on the market or in the clinic, although issues such as safety and applicability to the different types of epilepsy will have to be explored.

The approach, published in Nature Medicine, builds on previous work that separately linked epileptic seizures to disruption of the bloodbrain barrier $(\mathrm{BBB})^{2-4}$ and $\mathrm{CNS}$ inflammation to BBB disruption ${ }^{5,6}$ (see Figure 1, “Triggering a seizure”).

The University of Verona group and colleagues from four U.S. universities tested whether blocking leukocyte adhesion to brain blood vessel walls, a common pathway of CNS inflammation, could prevent epileptic seizures in mice.

In vivo staining and microscopy revealed that four different leukocyte adhesion molecules-intracellular adhesion molecule 1 (ICAM1; CD54), vascular cell adhesion molecule 1 (VCAM1; CD106), selectin E (SELE; CD62E) and selectin P (SELP; CD62P) - were upregulated on the brain endothelium following induction of seizure. The upregulation coincided with greater adhesion of leukocytes to cerebral blood vessel walls.
Figure 1. Triggering a seizure. Research published in Nature Medicine suggests that blocking inflammation pathways in the CNS vasculature could help treat or prevent recurrent seizures associated with epilepsy. ${ }^{1}$

[a] In a mouse model of pilocarpineinduced epilepsy, intraperitoneal delivery of pilocarpine resulted in upregulation of leukocyte adhesion molecules on brain vascular endothelial cells. Those molecules, which include vascular cell adhesion molecule 1 (VCAM1; CD106) and selectin $\mathrm{P}$, were then able to bind their corresponding receptors - such as integrin $\alpha_{4} \beta_{1}(\mathrm{VLA}-4)$ and selectin $P$ ligand (SELPLG; PSGL-1; CD162)-on circulating leukocytes, causing them to adhere to the vessel walls.

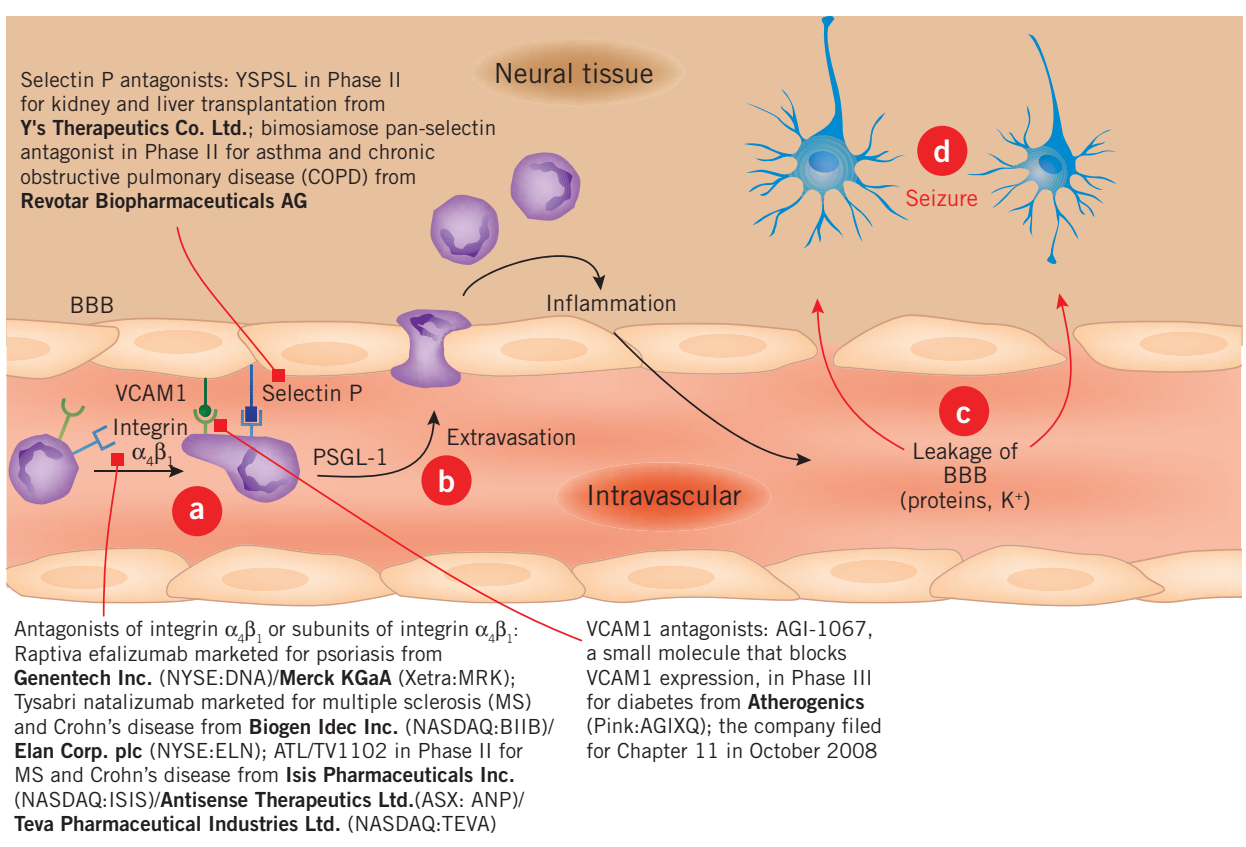

[b] Bound leukocytes then extravasated through the blood-brain barrier (BBB) and entered neural tissue, where they produced local inflammation, disrupting the BBB.

[c] Disruption of the BBB allowed leakage of plasma constituents such as proteins and excess potassium ions into neural tissue.

[d] Finally, the ion imbalance in the neural tissue helped contribute to neuronal hyperexcitability, generating a new seizure.

The authors suggest that blocking the initial interaction between leukocytes and adhesion molecules at step [a] could block BBB leakage and thus offer a new strategy for treating epileptic seizures that would not require a therapeutic to enter the brain. 
Antibodies targeting the adhesion molecules VCAM1 and SELP or their respective leukocyte receptors-integrin $\alpha 4$ subunit of the VLA4 receptor (ITGA4; CD49D) and selectin P ligand (SELPLG; PSGL-1; CD162)-decreased leukocyte adhesion to vessel walls compared with the effect of control antibody.

The anti-ITGA4 antibody produced the best results. Compared with what was seen in untreated control mice, the antibody significantly decreased recurrent spontaneous convulsions when given after seizure onset and prevented convulsions when given before seizure-inducing pilocarpine ( $p<0.001$ for both).

Postmortem tissue analysis of the mice confirmed that pilocarpine-induced opening of the $\mathrm{BBB}$ was lower in mice pretreated with the anti-ITGA4 antibody than it was in untreated mice, suggesting a link between leukocyte-vascular adhesions, BBB damage and seizure induction.

The authors concluded that "anti-adhesion therapies with humanized antibodies or other available approaches would allow clinical evaluation of adhesion pathways as targets for preventing epilepsy, particularly after inflammatory inciting events such as stroke or traumatic brain injury."

Other studies also have found that brain tissue isolated from some classes of epilepsy patients has high levels of inflammatory cells in cortical regions affected by seizures, noted Jacqueline French, a professor of neurology and director of the epilepsy clinical trials consortium at the New York University Comprehensive Epilepsy Center.

According to Richard Ransohoff, director of the Neuroinflammation Research Center at the Cleveland Clinic and staff neurologist at its Mellen Center for Multiple Sclerosis, the Nature Medicine paper offers a mechanism that potentially could be targeted by mAbs such as efalizumab or natalizumab, which are already on the market for inflammatory disorders like psoriasis, multiple sclerosis (MS) and Crohn's disease.

"Importantly, since the inflammatory mechanism occurs entirely on the blood side of the blood-brain barrier, the mAb would presumably not have to cross the BBB to show efficacy against seizures," said Ransohoff.

Raptiva efalizumab, which targets integrin $\alpha_{\mathrm{L}}$ (ITGAL; CD11a), is marketed by Genentech Inc. in the U.S. and by Merck KGaA elsewhere to treat plaque psoriasis. Tysabri natalizumab, which targets ITGA4, is marketed by Biogen Idec Inc. and Elan Corp. plc to treat MS in the EU and MS and Crohn's disease in the U.S.

Gabriela Constantin, who is assistant professor of pathology at the University of Verona and corresponding author on the paper, told SciBX she is also interested in testing selectin antagonists in the pilocarpineinduced epilepsy models and in other preclinical models of epilepsy.

At least two such antagonists are in the clinic. One is bimosiamose (TBC1269), a pan-selectin antagonist from Revotar Biopharmaceuticals AG, which is in Phase II testing to treat chronic obstructive pulmonary disease (COPD) and plaque psoriasis.

Another is YSPSL, a fusion protein of PSGL-1 and human IgG1 from Y's Therapeutics Co. Ltd. that acts as a selectin P antagonist. It has completed two Phase II trials: one to prevent delayed graft function in patients undergoing cadaveric kidney transplantation and one to prevent ischemic reperfusion injury in liver transplant patients.
Stefan Hemmerich, director of clinical operations at Y's, said company scientists are familiar with the Nature Medicine paper, which "may impact the company's strategy for developing follow-up indications for YSPSL beyond transplant." Indeed, he said, Y's is now considering a collaboration with Constantin and colleagues to evaluate YSPSL in preclinical epilepsy models.

\section{Seizing on inflammation}

Researchers polled by SciBX think targeting inflammation to treat or even prevent epileptic seizures has multiple advantages compared with targeting neuronal function.

According to French, those include "avoidance of side effects that can come with targeting neurons using anticonvulsants and the possibility of targeting a cause of the seizures and thus modifying disease."

Vincent Pieribone, associate professor of cellular and molecular physiology and neurobiology at Yale School of Medicine, said another advantage would be the prophylactic aspect of the strategy, "which could potentially help prevent the development of seizures in patients at risk of epilepsy, including victims of traumatic brain injury, stroke and cerebral infection."

He added, "The marketed anticonvulsant epilepsy drugs have little value in such a preventative context." (See Table 1, "Epilepsy pipeline.")

Pieribone is cofounder of Marinus Pharmaceuticals Inc. The company's lead compound is Ganaxolone, a synthetic analog of the neurosteroid allopregnanolone that targets the $\gamma$-aminobutyric acid $\alpha$-receptor (GABRA1; GABA receptor). Ganaxolone has completed two Phase II studies to treat epilepsy. Marinus is now looking for partners to help move the compound into Phase III testing, Pieribone told SciBX.

Matthew During, professor of molecular virology, immunology and medical genetics at Ohio State University, pointed out that the strategy might even be able to block seizures in specific regions of the brain, something not generally possible with standard epilepsy drugs.

"An epilepsy therapy with an ideal therapeutic index would be a compound that inhibits excessive excitatory activity and seizures in select areas of the brain without disrupting normal brain function. The marketed anticonvulsants fail to achieve that goal because they have a global inhibitory effect on the brain," he said.

During is cofounder of Neurologix Inc. The company's AAV-NPY, an adeno-associated virus (AAV) vector containing the neuropeptide $Y$ (NPY) gene that is stereotactically delivered to the brain, has shown efficacy in multiple rodent models of late-stage epilepsy. ${ }^{7}$ The company hopes to begin a Phase I trial this year in patients with drug-resistant temporal lobe epilepsy who are candidates for resective surgery.

\section{Other players}

At least two other research groups have identified additional inflammatory mediators that may contribute to BBB breakdown in epilepsy (see Box 1, "DELeting epilepsy").

Using a variation on the pilocarpine epilepsy model used in the Nature Medicine paper, a group led by Damir Janigro also found that leakage of 
Table 1. Epilepsy pipeline. At least nine compounds are in clinical development for epilepsy.

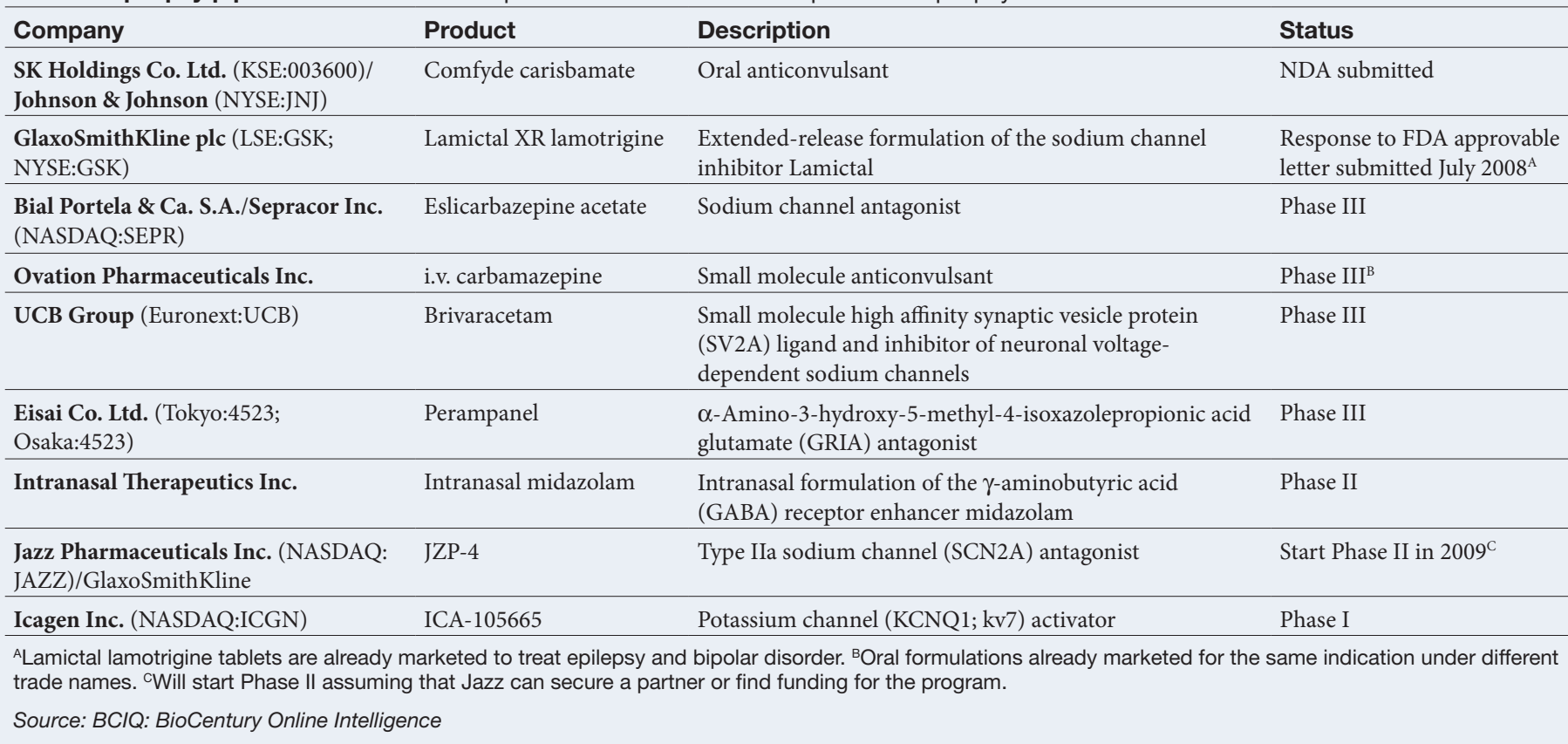

the BBB coincided with the occurrence of chemically induced seizures in mice. In addition, the researchers saw a rapid rise in serum levels of IL$1 \beta$, a known contributor to inflammation-mediated BBB leakage, prior to onset of seizure.

Pretreatment of the animals with antibodies targeting the IL-1 $\beta$ receptor lowered the frequency and intensity of seizures and decreased leakage of the BBB compared with what was seen in saline-treated controls. ${ }^{8}$

Janigro, a professor of molecular medicine and director of the Center for Cerebrovascular Research at the Cleveland Clinic, told SciBX he has applied for a patent covering the use of an intravenously or intraarterially delivered IL- $1 \beta$ receptor antagonist to treat or prevent acute epileptic seizures and other neurological disorders caused by inflammatory disruption of the BBB.

Amgen Inc. and Biovitrum AB market Kineret anakinra, a recombinant IL-1 receptor antagonist (IL1RN; IL1RA), to treat rheumatoid arthritis (RA).

"Mounting evidence from our own lab and others suggests that inflammatory events at the blood-brain barrier may indeed cause the initial seizures that lead to rewiring of brain circuitry and the development of epilepsy," said Janigro. "In other words, epilepsy likely has a key cerebrovascular aspect just as important as the neuronal aspect that has always been recognized."

The good news, he said, is that serum markers might exist to help identify epilepsy patients who are at increased risk of inflammatory BBB breakdown. These patients could benefit from an anti-inflammatory therapy in addition to anticonvulsants.

"Indeed," Janigro noted, "traditional anti-inflammatory steroids are already used sometimes to treat epileptic seizures in an emergency setting and epileptic seizures in infants."

Preclinical work by Alon Friedman and colleagues at the Ben-Gurion University of the Negev has shown that targeting transforming growth factor- $\beta$ receptor 1 (TGFBR1; ALK5) at the BBB could potentially help decrease the likelihood of developing epilepsy and other forms of cortical dysfunction following trauma. ${ }^{9}$

They found that exposing rat brains to the BBB-disrupting agents deoxycholic acid or a concentrated serum albumin solution resulted in vasogenic edema and epileptic seizures. Brain slices from rats exposed to albumin in the presence of a TGFBR1 antagonist showed significantly less seizure activity than brain slices from rats exposed only to albumin $(p<0.001)$.

\section{Testing more models}

A key challenge in translating the link between inflammation and epilepsy into the clinic will be identifying appropriate patient populations. A first step in that direction could include looking at a wider variety of animal models.

"A rodent model of chronic spontaneous seizures would offer a better proxy for late-stage epilepsy in humans than the pilocarpine model of acute seizure used in the paper," said During. "Looking at the anti-inflammatory strategy in two other rodent models of adult-onset epilepsypost-traumatic epilepsy and glioma-related epilepsy-might provide further indication of how generally the strategy can treat epilepsy."

Those other models could help determine whether the Verona approach can minimize epilepsy progression when intervening at later stages of disease, according to Jan Gorter, a researcher in the Swammerdam Institute for Life Sciences at the University of Amsterdam. Gorter said the models might also be useful in figuring out whether stopping 


\section{Box 1. DELeting epilepsy.}

NIH researchers have identified an endogenous inhibitor of leukocyte adhesion, DEL1, that could provide a starting point for designing anti-leukocyte adhesion therapies to treat CNS inflammation associated with epilepsy and other disorders. ${ }^{11}$

In a paper in Science, the group showed that increased expression of the protein DEL1 (EGF-like repeats and discoidin I-like domains 3; EDIL3) could lower inflammation in mice. Del1 ${ }^{-/-}$mice had greater neutrophil recruitment in the bronchoalveolar lavage fluid than wild-type controls that also received lipopolysaccharide (LPS) challenge, suggesting that DEL1 represses recruitment of inflammatory compounds.

In the same Del1 knockout mice, the team knocked out lymphocyte function-associated antigen 1 (CD11a-CD18; LFA-1), which mediates leukocyte adhesion to endothelial cells during inflammation. There was no significant difference in neutrophil recruitment between the double-knockout and control mice. This suggested that the increased inflammatory response caused by DEL1 knockout is dependent on LFA-1.

Finally, cellular adhesion assays showed that DEL1 inhibited LFA-1-dependent leukocyte adhesion to endothelial cells.

The findings open a new therapeutic avenue for DEL1. The molecule was previously studied in the cardiovascular space. In 2004, Deltavasc, a cationic lipid for delivery of the DEL1 gene from
Valentis Inc. (now Urigen Pharmaceu-

ticals Inc.), failed to meet the primary endpoint in a Phase II trial to treat peripheral arterial disease (PAD).

Triantafyllos Chavakis, head of the inflammation biology section and investigator at the NIH's National Cancer Institute as well as lead author on the paper, told SciBX it will be necessary to determine the minimal part of DEL1 that has inhibitory activity in order to develop compounds with an improved half-life compared with that of the full-length protein.

The effects of DEL1 or its minimal inhibitory component would then need to be tested in animal models of inflammation, he said.

Alternatively, Björn Frendéus, senior principal scientist of preclinical research at Biolnvent International AB, said a first step in the development of a therapeutic could be proof of concept for a DEL1 fusion protein, such as a DEL1-IgG Fc. "Such a fusion construct will provide DEL1 with a longer terminal half-life whilst hopefully retaining the low immunogenicity of the native DEL1 protein," he said.

Biolnvent 's $\mathrm{BI}-505$ is an anti-intracellular adhesion molecule 1 (ICAM1; CD54) antibody in preclinical development for cancer. ICAM1 interacts with LFA-1 to facilitate leukocyte adhesion.

Companies targeting LFA-1 include Eli Lilly and Co., which has an LFA-1 antagonist in preclinical testing to treat psoriasis, and SARcode Corp. and Sunesis Pharmaceuticals Inc., which have SAR1118 in Phase I testing for conjunctivitis. Cerep S.A. and Laboratoires Thea S.A. are co-developing a topically formulated LFA-1 receptor antagonist for ophthalmic indications. Clinical development of an oral version of the compound for systemic inflammatory disorders was previously stopped because of hepatotoxicity after oral delivery.

Frendéus cautioned that DEL1 also has been proposed to regulate other cellular processes, including angiogenesis, through interactions with different integrin family receptors. Therefore, he said, it will be important to understand the mode of action of a DEL1-based construct.

"The reported ability of DEL1 to bind and modulate not only integrin $\beta_{2}-$ dependent cell adhesion processes, but also other cellular processes, needs to be carefully addressed from a safety and tolerability point of view," said Frendéus. "However, if a DEL1-based therapeutic construct could be engineered to both block inflammatory cell recruitment and reduce pathological angiogenesis, it might be a new blockbuster."

According to the patent and licensing departments at the $\mathrm{NIH}$, a patent application covering the research has been filed, and the use of these findings is available for licensing.

- Lauren Martz, Staff Writer treatment causes exacerbation of disease, as can occur in animal models of MS.

It might turn out that the strategy works only in a short window after initial trauma, because multiple injury-related molecular cascades are likely activated at later time points. Thus, a modified strategy combining anti-inflammatory agents with compounds that decrease neurodegeneration might be one approach to preventing seizures or seizure progression in later-stage epilepsy, Gorter noted.

"Studies in larger mammals, perhaps dogs or monkeys, would also be a useful next step to test the anti-inflammatory strategy in brains that more closely approximate the volume and vasculature of the human brain," said Pieribone.
Constantin told SciBX that she and colleagues are already looking at other epilepsy animal models, including a pentylenetetrazole model of convulsions, a kainic acid-induced epilepsy model and an electrical field model.

In terms of the appropriate patient population, a key challenge is that there is no indication that inflammation and related BBB breakdown is a key characteristic of all forms of epilepsy. "In multiple sclerosis there is extensive inflammation and BBB breakdown at specific locations, and yet most MS patients don't have epilepsy," Gorter noted.

Thus, said French, "it will be important to have a means for identifying appropriate patient populations. That could involve peripheral serum blood tests for markers and perhaps diagnostic imaging techniques." 
Along those lines, Friedman and colleagues have already used brain MRI to compare head trauma patients with and without post-traumatic epilepsy and found that patients with epilepsy had higher BBB permeability than nonepileptic patients. ${ }^{10}$

Constantin said her lab is studying inflammatory parameters in the blood and the brains of epilepsy patients at different time points after seizures.

In terms of safety, experience with MS patients could be useful.

"It's critically important to look at the safety profile of chronic use of anti-inflammatory mAbs in the context of what we've learned from treating inflammatory diseases such as MS," said Ransohoff. "That should help clarify any potential challenges associated with repurposing an immunosuppressive drug to epilepsy."

A promising antibody eventually might be tested in a small proofof-principle trial in patients at risk of seizure, he added, "with rigorous monitoring for adverse events like opportunistic infection."

At Y's Therapeutics, Hemmerich noted that the company's YSPSL selectin $\mathrm{P}$ antagonist already has shown a good safety profile in about 700 patients to date, which could expedite its clinical development in neurological disorders if the compound shows efficacy in preclinical epilepsy models.

The authors on the Nature Medicine paper have applied for a U.S. patent covering the use of an antagonist of leukocyte recruitment or trafficking to prevent or treat recurrent seizures and epilepsy.

Fulmer, T. SciBX 2(1); doi:10.1038/scibx.2009.1

Published online Jan. 8, 2009

\section{REFERENCES}

1. Fabene, P. et al. Nat. Med.; published online Nov. 23, 2008; doi:10.1038/nm.1878

Contact: Paolo Fabene, University of Verona, Verona, Italy e-mail: paolo.fabene@univr.it

Contact: Gabriela Constantin, same affiliation as above e-mail: gabriela.constantin@univr.it

2. Marchi, N. et al. Epilepsia 48, 732-742 (2007)
3. Van Vliet, E.A. et al. Brain 130, 521-534 (2007)

4. Seiffert, E. J. Neurosci. 24, 7829-7836 (2004)

5. Lucas, S.-M. et al. Br. J. Pharmacol. 147, S232-S240 (2006)

6. Ransohoff, R. et al. Nat. Rev. Immunol. 3, 569-581 (2003)

7. Noe, F. et al. Brain 131, 1506-1515 (2008)

8. Marchi, et al. Neurobiol. Dis.; published online Oct. 28, 2008; doi:10.1016/ j.nbd.2008.10.002

9. Ivens, S. et al. Brain 130, 535-547 (2007)

10. Tomkins, O. et al. J. Neurol. Neurosurg. Psychiatry 79, 774-777 (2008)

11. Choi, E. et al. Science; published online Nov. 13, 2008; doi:10.1126/science.1165218

Contact: Triantafyllos Chavakis, National Institutes of Health, Bethesda, Md.

e-mail: chavakist@mail.nih.gov

COMPANIES AND INSTITUTIONS MENTIONED

Amgen Inc. (NASDAQ:AMGN), Thousand Oaks, Calif.

Ben-Gurion University of the Negev, Beer-Sheva, Israel

Biogen Idec Inc. (NASDAQ:BIIB), Cambridge, Mass.

Biolnvent International AB (SSE:BINV), Lund, Sweden

Biovitrum AB (SSE:BVT), Stockholm, Sweden

Cerep S.A. (Euronext:CER), Paris, France

Cleveland Clinic, Cleveland, Ohio

Elan Corp. plc (NYSE:ELN), Dublin, Ireland

Eli Lilly and Co. (NYSE:LLY), Indianapolis, Ind.

Food and Drug Administration, Rockville, Md.

Genentech Inc. (NYSE:DNA), South San Francisco, Calif.

Laboratoires Thea S.A., Clermont Ferrand, France

Marinus Pharmaceuticals Inc., Branford, Conn.

Merck KGaA (Xetra:MRK), Darmstadt, Germany

National Cancer Institute, Bethesda, Md.

National Institutes of Health, Bethesda, Md.

Neurologix Inc. (OTCBB:NRGX), Fort Lee, N.J.

New York University, New York, N.Y.

Ohio State University, Columbus, Ohio

Revotar Biopharmaceuticals AG, Berlin, Germany

SARcode Corp., San Francisco, Calif.

Sunesis Pharmaceuticals Inc. (NASDAQ:SNSS),

South San Francisco, Calif.

University of Amsterdam, Amsterdam, the Netherlands

University of Verona, Verona, Italy

Urigen Pharmaceuticals Inc. (OtcBB:URGP), Burlingame, Calif.

Y's Therapeutics Co. Ltd., Tokyo, Japan

Yale School of Medicine, New Haven, Conn. 